\title{
Modulations of neural activity in auditory streaming caused by spectral and temporal alternation in subsequent stimuli: a magnetoencephalographic study
}

\author{
Ivan Chakalov ${ }^{1 \dagger}$, Rossitza Draganova ${ }^{2}$, Andreas Wollbrink $^{1}$, Hubert Preiss| ${ }^{2}$ and Christo Pantev ${ }^{1 *}$
}

\begin{abstract}
Background: The aim of the present study was to identify a specific neuronal correlate underlying the preattentive auditory stream segregation of subsequent sound patterns alternating in spectral or temporal cues. Fifteen participants with normal hearing were presented with series' of two consecutive ABA auditory tone-triplet sequences, the initial triplets being the Adaptation sequence and the subsequent triplets being the Test sequence. In the first experiment, the frequency separation (delta- $f$ ) between A and B tones in the sequences was varied by 2 , 4 and 10 semitones. In the second experiment, a constant delta-f of 6 semitones was maintained but the InterStimulus Intervals (ISIs) between A and B tones were varied. Auditory evoked magnetic fields (AEFs) were recorded using magnetoencephalography (MEG). Participants watched a muted video of their choice and ignored the auditory stimuli. In a subsequent behavioral study both MEG experiments were replicated to provide information about the participants' perceptual state.
\end{abstract}

Results: MEG measurements showed a significant increase in the amplitude of the B-tone related P1 component of the AEFs as delta-f increased. This effect was seen predominantly in the left hemisphere. A significant increase in the amplitude of the N1 component was only obtained for a Test sequence delta-f of 10 semitones with a prior Adaptation sequence of 2 semitones. This effect was more pronounced in the right hemisphere. The additional behavioral data indicated an increased probability of two-stream perception for delta-f $=4$ and delta-f $=10$ semitones with a preceding Adaptation sequence of 2 semitones. However, neither the neural activity nor the perception of the successive streaming sequences were modulated when the ISIs were alternated.

Conclusions: Our MEG experiment demonstrated differences in the behavior of P1 and N1 components during the automatic segregation of sounds when induced by an initial Adaptation sequence. The P1 component appeared enhanced in all Test-conditions and thus demonstrates the preceding context effect, whereas N1 was specifically modulated only by large delta-f Test sequences induced by a preceding small delta-f Adaptation sequence. These results suggest that $\mathrm{P} 1$ and $\mathrm{N} 1$ components represent at least partially-different systems that underlie the neural representation of auditory streaming.

\footnotetext{
* Correspondence: pantev@uni-muenster.de

${ }^{\dagger}$ Equal contributors

${ }^{1}$ Institute for Biomagnetism and Biosignalanalysis, University of Münster,

Malmedyweg 15, 48149, Münster, Germany

Full list of author information is available at the end of the article
} 


\section{Background}

The brain's ability to constantly organize auditory objects or "auditory streams" from the competing sounds in the environment is a key element in human auditory perception. This phenomenon has been labeled "stream segregation" or "streaming" by Bergman and Campbell [1,2]. The most commonly-used stimulus configuration for investigating 'streaming', as described by Van Noorden [3], consists of a low-frequency tone A and high-frequency tone $B$ presented in a sequence of repeated $\mathrm{ABA}$ triplets. When the frequency separation $(\Delta \mathrm{f})$ between the tones is small and the inter stimulus interval (ISI) is long, the sequence is typically heard as one sound stream, like a gallop. In the case of a large $\Delta f$ and brief ISI the galloping rhythm is no longer heard and, instead, the perception switches to two distinct sound streams of A and B tones [3,4]. At intermediate $\Delta \mathrm{f}$ and ISI values, the perception could bias in favor of one stream or two streams, depending on participants' attention [3,5] and the duration of listening [6].

According to the "peripheral channeling hypothesis", streaming occurs when different sounds excite adjacent peripheral channels with minimal overlapping in the cochlea and the auditory nerve [7]. However, the existence of additional acoustic cues that influence perceptual organization $[6,8-11]$ as a function of time [2,6], suggest the contribution of centrally computed features in stream segregation [12-28]. An interaction between sub-cortical and cortical auditory structures in switching perception from non-streaming to streaming has been demonstrated recently [29].

Most electrophysiological studies interpret the neural correlates of streaming in terms of tonotopic organization of the auditory system [5,7,12,14,15,17-19,30-32], and Elhilali and colleagues demonstrated the critical role played by temporal coherence in the formation of auditory streams [33]. They showed that frequency-distant tones are no longer heard as distinct sound streams if presented synchronously rather than successively, although the enhanced neuronal evoked activity ascribed both sequences (synchronous and asynchronous) to streaming [33]. Thus, tonotopic organization itself is not enough to explain this type of perception; streaming requires both frequency separation and temporal incoherence [33]. In real acoustic environments, such as music and speech, however, sounds appear successively, and the perception and corresponding evoked activity of streaming are highly influenced by recent auditory experience $[31,34,35]$. Furthermore, the encoding of subsequent sounds can be influenced by attention [36,37] and distinct neuronal mechanisms are responsible for the automatic segregation of sounds and attention-dependent build-up processes $[8,22,28,38]$.
The present study aimed to investigate the neural bases of auditory streaming at a pre-attentive level by making alterations to the spectral or temporal features of succeeding sound sequences. Our experiments were motivated by the hypotheses that selective adaptation underlies stream segregation $[15,16,23]$ and that modulations in auditory evoked activity can indicate this process [16,21,22,35,39]. Since streaming is based on adaptation to specific tone frequencies [14-16] and is strongly determined by the ISI [40], it seems reasonable to expect the encoding of subsequent streaming patterns to rely on similar physiological mechanisms. Furthermore, multiple levels of representation in auditory stream segregation have been proposed by psychophysical comparisons of the effects of different types of initial adaptation stimuli $[23,34]$. Neuronal activity in streaming is also known to be modulated by prior perception $[28,35]$. In an event-related potential study by Sussman \& Steinschneider, for instance, they showed the absence of mismatch negativity to a 'deviant tone' (a tone which deviated in frequency) from their repeating ABBB Test sequence induced by a priming sequence [28]. Neurological responses to the deviant tones are thought to arise during streaming and thus the authors concluded that no streaming occurred when the Test sequence was preceded by a smaller $\Delta \mathrm{f}$ Adaptation sequence. No behavioral responses were collected, however, so comparison of behavioural reponses with evoked activity was not possible[28]. Another EEG research by Snyder and colleagues found that the adaptation to a prior stimulus pattern could underline the effects of functionally distinct neural processes in stream segregation [35]. They also demonstrated that a large $\Delta \mathrm{f}$ in the initial Adaptation sequence results in reduced streaming on the subsequent Test sequence and vice versa [35].

The current study tested the hypotheses that frequency-selective adaptation underlies stream segregation and that prior adaptation stimulation could recover specific neural correlates and streaming effects. We measured the auditory evoked fields (AEF) in response to an ABA triplet paradigm consisting of successive prime Adaptation and subsequent Test sequences. The contextual effect between consecutive tone patterns is known to decrease as the period of silence between them increases $[2,31,35,40,41]$, therefore the neurophysiological demonstration of a clear adaptation effect requires continual stimulation. For this reason we chose to present our experimental conditions as an uninterrupted trial (without silence between the sequences). In order to distinguish between adaptation-based effects and those modulated by attention, the participants were instructed to concentrate on a soundless movie of their choice and ignore the ongoing auditory stimulation. We set up two consecutive MEG experiments to manipulate 
either the frequency separation $(\Delta \mathrm{f})$ or the inter stimulus interval (ISI) of the succeeding adaptation and test sequences. In addition, behavioral measurements were performed, whilst replicating all sessions of the MEG study, in order to evaluate the participants' perceptual state.

In the first experiment, $\Delta \mathrm{f}$ between the $\mathrm{A}$ and $\mathrm{B}$ tones of the initial Adaptation and subsequent Test sequences was altered (small, intermediate or large $\Delta f$ ) by varying the frequency of the $\mathrm{B}$ tones. The frequency of the $\mathrm{A}$ tones did not vary throughout the entire experiment. We anticipated that the presentation of a small $\Delta \mathrm{f}$ during the Adaptation sequence would induce increased neuronal activity during a subsequent intermediate or large $\Delta \mathrm{f}$ Test sequence because of the concomitant reduction in neuronal adaptation as $\Delta \mathrm{f}$ increases at the beginning of the Test sequence. We also expected to see the complimentary result, that large and intermediate $\Delta f$ values during the Adaptation sequence would result in decreased neuronal activity during the small $\Delta \mathrm{f}$ Test sequence. The ISI between A and B tones did not vary during this first trial.

In the second experiment, a constant $\Delta \mathrm{f}$ of 6 semitones was used and the ISI was varied. A short ISI in the Adaptation sequence was followed by a long ISI in the Test sequence and vice versa. In this experiment we specifically investigated whether the undergoing adaptation effect is dependent upon the specific frequency of the A or B tone, or whether particular neurons might be tuned to a specific $\Delta \mathrm{f}$-shift, regardless of the actual frequency range $[2,3]$. The process of adaptation in response to simple frequencies in the auditory system is known to be dependent on the concrete repetition rate of the stimuli
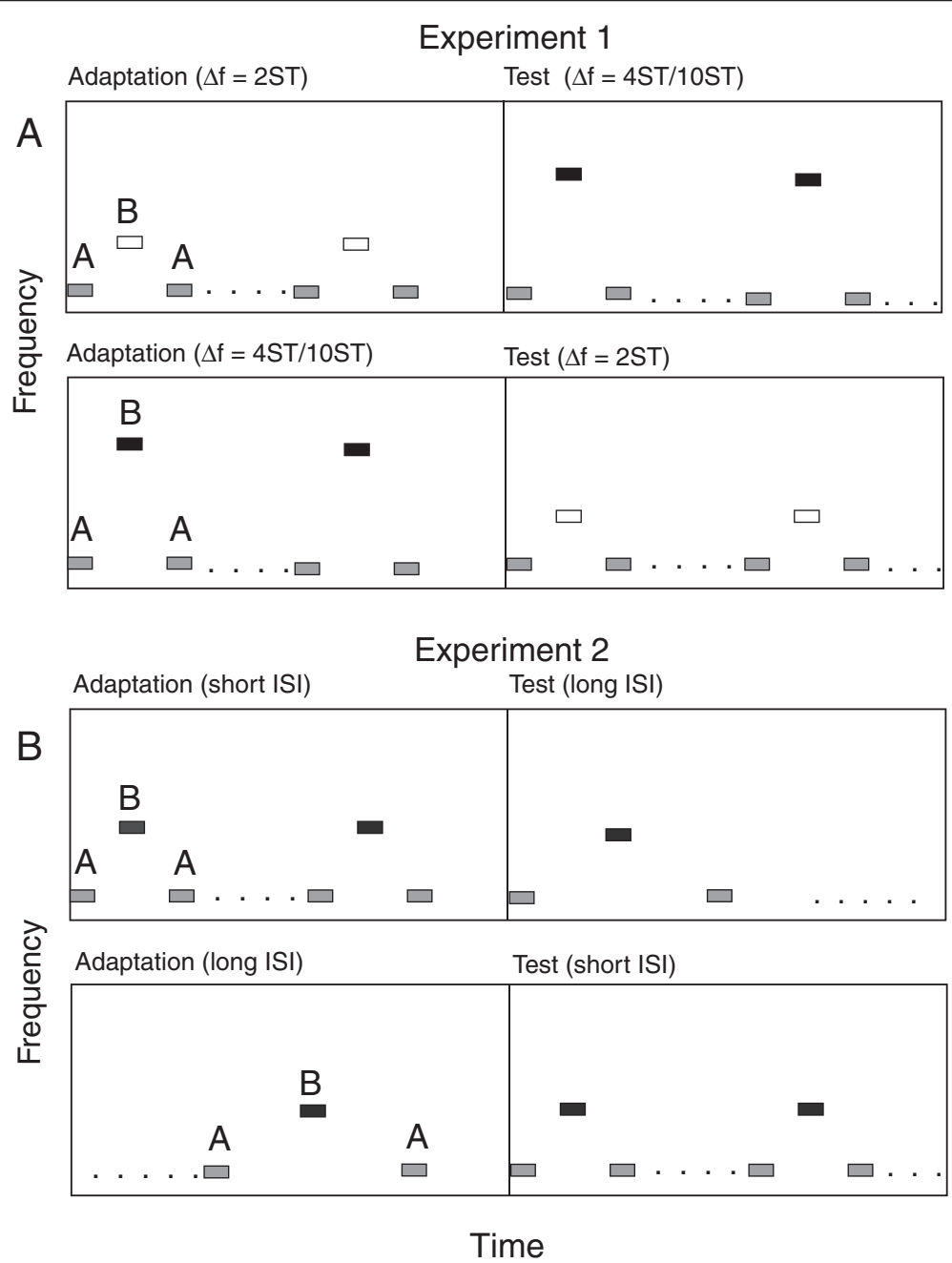

Figure 1 Experimental design. This figure illustrates the two different ABA sequences (Adaptation and Test) presented in Experiments 1 and 2 , plotted as relative Frequency against relative Time. These sequences were presented with randomly-organized changes in position in uninterrupted trials. (A) Experiment-1; The Inter Stimulus Interval (ISI) between A and B tones in both sequences was fixed at $125 \mathrm{~ms}$. Three different degrees of $\Delta f(2,4$ and 10 semitones) were presented as either Adaptation or Test sequence. (B) Experiment-2; $\Delta f$ between successive $A$ and $B$ tones was fixed at 6 semitones but the ISI varied between short (100 ms) and long (225 ms). 
$[15,16]$. Thus, if the expectations proposed above (for Experiment 1 ) rely on adaptation to the repeated presentation of the $\mathrm{A}$ and $\mathrm{B}$ tones, changing the rate of presentation between the two sequences should disrupt the adaptation course and not induce modulation of the neuronal activity during the Test-sequence in that experiment.

\section{Methods}

\section{Test participants}

Fifteen right-handed participants ( 5 males) aged between 22 and 30 years participated in this study. None of them had a history of otological or neurological disorders. Normal audiological status, defined by air conduction hearing thresholds of less than $10 \mathrm{~dB}$ HL in the frequency range between 250 and $4000 \mathrm{~Hz}$ was verified by pure tone audiometry. Participants gave written informed consent to take part in the study in accordance with procedures approved by the Ethics Commission of the University of Münster and the Declaration of Helsinki.

\section{MEG experiments}

Two different MEG experiments were carried out using ABA tone-pip sequences (sinusoidal tone-pips of $25 \mathrm{~ms}$ duration, including $10 \mathrm{~ms}$ rise and decay times). The loudness of the stimuli was set to $60 \mathrm{~dB}$ above the individual hearing thresholds. Trials were organized as a combination of the Adaptation sequence and subsequent Test sequence in an uninterrupted trial. In two subsequent experiments we investigated the effects of the preceding Adaptation sequence on the following Test sequence by varying (a) frequency separation (Experiment 1) and (b) inter stimulus interval (Experiment 2) (c.f. Figure 1). The duration of each trial was $12 \mathrm{~s}: 6 \mathrm{~s}$ for the adaptation and $6 \mathrm{~s}$ for the following test sequence. In each experiment, 80 trials were presented: 40 Adaptation sequences and 40 Test sequences. The ordering of the Adaptation and Test sequences was randomly organized. The inter trial interval (ITI) was $3 \mathrm{~s}$, thus the total recording time of one experimental session was 20 minutes.

Experiment 1 consisted of two 20-minute sessions during which three different $\Delta \mathrm{f}$ values for the ABA triplets were presented: small (2 semitones), intermediate (4 semitones) and large (10 semitones). In each case the frequency of tone A was $500 \mathrm{~Hz}$; therefore tone B was either $561 \mathrm{~Hz}$ for the small $\Delta \mathrm{f}, 630 \mathrm{~Hz}$ for the intermediate $\Delta \mathrm{f}$ or $891 \mathrm{~Hz}$ for the large $\Delta \mathrm{f}$, respectively. The ISI linking the ABA tones as triplets was constantly set to $100 \mathrm{~ms}$; thus the ISI between successive A tones was $225 \mathrm{~ms}$ and between successive B tones was $475 \mathrm{~ms}$.

In the first session a small $\Delta \mathrm{f}$ Adaptation sequence was followed by a intermediate $\Delta \mathrm{f}$ Test sequence and vice versa (c.f. Figure 1A), with the exact ordering of these conditions randomized. The second session compared small $\Delta \mathrm{f}$ Adaptation with large $\Delta \mathrm{f}$ Test sequences, and vice versa, in the same way.

Experiment 2 consisted of one 20-minute session in which the ISIs between A and B tones in consecutive sequences were changed, opposing long ISI vs. short ISI and vice versa (c.f. Figure $1 B$ ). $\Delta$ f remained at 6 semitones $(\mathrm{A}=500 \mathrm{~Hz}$ and $\mathrm{B}=707 \mathrm{~Hz}$ ) throughout Experiment 2, a value that has been previously determined in many studies to facilitate the perception of two streams [16,21,22,34,35]. In the long ISI condition, the period between the ABA triplet tones was $225 \mathrm{~ms}$; thus, the ISI of successive A tones and successive $B$ tones remained at $475 \mathrm{~ms}$ and $975 \mathrm{~ms}$, respectively. In the short ISI condition, the A and B tones were presented twice as fast, with an ISI of $100 \mathrm{~ms}$ between triplet tones. The ISIs between successive A and successive $B$ tones were therefore $225 \mathrm{~ms}$ and $475 \mathrm{~ms}$, respectively. In the same manner as in Experiment 1, a short ISIAdaptation sequence was followed by a long ISI-Test sequence and vice versa (long ISI followed by a short ISI), with the exact ordering of these conditions randomized.

\section{Behavioral experiment}

In the behavioral experiment we used the same experimental design as the MEG measurements (described above, also c.f. Figure 1) to investigate the participants' perceptual state. Three participants from the prior MEGmeasurements could not participate in the behavioral tests. Three sessions were carried out, corresponding to both MEG-experiments. In the first session a small $\Delta f$ Adaptation sequence was followed by a intermediate $\Delta \mathrm{f}$ Test sequence and vice versa, in randomized order (as in session one of Experiment 1). The second session compared small $\Delta \mathrm{f}$ Adaptation with large $\Delta \mathrm{f}$ Test sequences, and vice versa (as in session two of Experiment 1). In the third behavioral session a short ISI Adaptation sequence was followed by a long ISI Test sequence and vice versa (as in Experiment 2). All three 15 minute sessions took place on the same day two weeks after the MEGmeasurements. Within each session 24 trials were presented, corresponding to 12 repetitions of each of the conditions (small versus intermediate $\Delta \mathrm{f}$; small versus large $\Delta \mathrm{f}$; short versus long duration ISI). In order to avoid the effect of "echoic memory" involving successive trials of active listening [34,42-44], the trial duration was shortened to $8 \mathrm{~s}$ (4s of Adaptation and $4 \mathrm{~s}$ of Test) and the inter trial interval (ITI) was extended to $5 \mathrm{~s}$. The participants were instructed to indicate the point at which their perception switched from one to two streams by pressing a mouse button during the presentation of each sequence. Prior to the experiment, the participants practiced 4 sequences of each condition in order to restrict intentional biasing of the perception in favor of one of the streams (A or B tones) [3,5]. The responses to each 
condition in each position as Adaptation or Test sequence were collected separately across all three sessions.

\section{MEG data acquisition}

The MEG recording was performed using a 275-channel whole-head system (Omega2005, VSM-Medtech, Port Coquitlam, BC, Canada), sampled at $600 \mathrm{~Hz}$. The MEG measurements preceded the behavioral recordings in order to avoid possible effects of attention, intention and prior experience. The participants had not received prior information about the paradigm, the stream segregation phenomena or the purpose of the study. They were comfortably seated in an upright position, instructed to ignore the sounds and watch a soundless video of their choice, presented on a monitor in front of them [16,22]. Eighty trials per session were recorded, yielding a sufficient number of MEG data.

\section{Data analysis of MEG data}

The MEG analysis was performed using the CTF software package. After applying a high-pass filter with cutoff-frequency of $1 \mathrm{~Hz}$ the MEG data were separated into epochs of $450 \mathrm{~ms}$ (from -50 to $+400 \mathrm{~ms}$ relative to the stimulus onset of the ABA triplets). Epochs with magnetic field amplitudes (peak-to-peak) larger than $3 \mathrm{pT}$ were excluded from further analysis as artifacts. After averaging, a $20 \mathrm{~Hz}$ low-pass filter was applied. Importantly, the responses to the first and last triplets in each condition were excluded from the averaging procedure in order to avoid a stronger influence from the onset and offset of each sequence on the grand average data.

Assuming the model of an equivalent current dipole (ECD) in a spherical volume conductor, a spatiotemporal dipole fit was performed. T1-weighted magnetic resonance imaging (MRI) of the head was obtained from all listeners on a $3 \mathrm{~T}$ Scanner (Gyroscan Intera T30, Philips, Amsterdam, Netherlands). The parameters (center location and radius) of the spherical head model were derived from the individual MRI. The interval used for the ECD fit ( $30 \mathrm{~ms})$ was placed around the local maximum of the P1 component of the AEF. The P1 dipolar sources evoked by tone B were less variable across conditions compared to the N1 sources and, thus, provided a better signal-to-noise ratio. For each participant, two ECDs (one in each hemisphere) were determined, defined by their dipole moment, orientation and spatial coordinates (a goodness of fit larger than 85\% was imposed). Median values of $\mathrm{x}, \mathrm{y}$, and $\mathrm{z}$ coordinates of the dipole location and the angles of the dipole orientation were calculated for each condition. The individual averaged values for P1 (across all conditions) were used to fix the dipole position and orientation and then the source space projection method [45] was applied to calculate the components of the transient evoked response (P1, N1) for each condition.

\section{Statistical analysis of MEG data}

The major goal of this study was to examine the influence of the initial stimulation on the Test sequence condition. For that purpose, the responses of the same condition presented in Adaptation and Test positions were compared (cross-checking comparison).

Repeated measurements 2x2x2 ANOVA were performed for the peak amplitudes and latencies of $\mathrm{P} 1$ and N1 components to the B tones of the ABA triplet. The statistical analysis always included three factors: "Hemisphere" (right $(\mathrm{RH})$ and left $(\mathrm{LH})$ ), " $\Delta \mathrm{f}$ " (Experiment 1) or "ISI" (Experiment 2) consisting of 2 variables for each of the investigated $\Delta \mathrm{f}$ values $(\Delta \mathrm{f}=2$ vs. $\Delta \mathrm{f}=4$ or 10 semitones, or short ISI vs. long ISI) and "Part" (also containing two variables, showing that the stimulus was presented as an Adaptation or Test condition). For two participants, P1 (one participant) and/or N1 components (one participant) could not be derived from the average response time course, and were not included in the group analysis.

Four separate ANOVAs were performed for Experiment 1 and one further separate ANOVA for Experiment 2. For all statistical analyses Bonferroni correction was applied.

In the first analyses the 2 and 4 semitone conditions from the first session of Experiment 1 were examined. This enabled us to test the effect of the initial 4 semitones condition on the subsequent 2 semitones Test and the effect of the initial 2 semitones on the 4 semitones Test by crosschecking the effects of the identical condition presented in Adaptation and Test positions. Another separate ANOVA was applied for the second session, to investigate the effect of 2 semitone Adaptations on the 10 semitone Test and vice versa. In the third analyses, the initial effect of the 2 semitone conditions on the 4 and 10 semitone Tests was examined. For that purpose, the 4 and 10 semitone conditions from the first and second sessions, presented in both Adaptation (for control) and Test positions were entered into another ANOVA. In a fourth ANOVA only the 2 semitones Test conditions from session one (following an initial 4 semitones) and session two (initial 10 semitones) of Experiment 1 were entered. In that way the initial effects of 4 and 10 semitone Adaptation conditions on the neural activity of the 2 semitone Test were investigated. In this analysis " $\Delta$ - $\mathrm{f}$ " was labeled "session" (2 semitone condition from session 1 and from session 2).

A separate ANOVA-analysis was also used for Experiment 2 , in the same way as for the first experiment. 


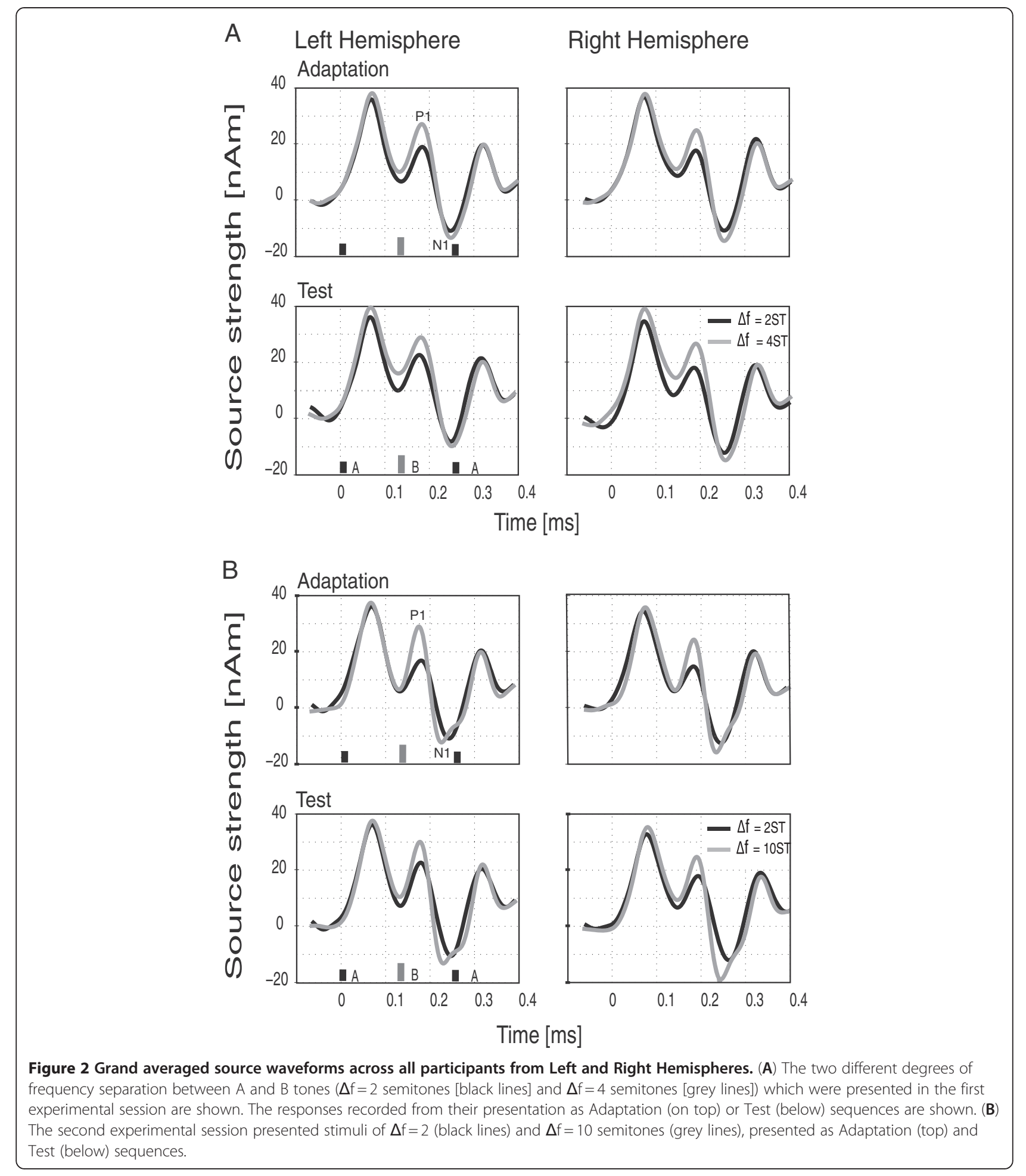

\section{Results}

\section{Source waveform data}

Experiment 1. Frequency-based neuronal activity and lateralization effects

The grand averaged responses from the first session of Experiment 1, are shown in Figure 2A (small $\Delta \mathrm{f}[2$ semitones] are indicated by black lines; intermediate $\Delta \mathrm{f}$ [4 semitones] are in gray). The upper and the lower graphs display the responses to the Adaptation and Test sequences, respectively. The amplitude of the P1 component was generally found to have increased for $\Delta f=4$ semitones compared to $\Delta \mathrm{f}=2$ semitones (significant 
main effect $\Delta f[F(1,14)=23.468, p<.001])$, whereas the amplitude of the N1 component was only slightly stronger in $\Delta \mathrm{f}=4$ semitones compared to $\Delta \mathrm{f}=2$ semitones. The P1 amplitudes were also slightly larger in the left compared to the right hemisphere. In contrast, the N1 amplitude was significantly enhanced in both conditions ( 4 and 2 semitones) in the right hemisphere compare to left hemisphere only on a Test position (interaction Hemisphere x Part for N1 amplitude $[F(1,14)=13.993$, $p<.05])$.

Figure $2 \mathrm{~B}$ shows the grand average responses from the second session of Experiment 1, with the P1 component significantly stronger in condition $\Delta \mathrm{f}=10$ semitones compared to the $\Delta \mathrm{f}=2$ semitones (significant main effect $\Delta \mathrm{f}[F(1,13)=12.598, p<.001])$. This enhanced amplitude was more prominent when presented as a Test sequence (main effect "Part" for P1 amplitude $[F(1,13)=5.610, p$ $<.05]$ ). The N1 amplitude was also significantly enhanced when the 10 semitones condition was presented as a Test sequence and it was more dominant in the $\mathrm{RH}$ (significant interaction $\Delta \mathrm{f} \mathrm{x}$ Part for $\mathrm{N} 1$ amplitude $[F(1,13)=8.384, p<.05]$ and main effect of Hemisphere $[F(1,13)=8.542, p<.05])$. In addition, the N1 was significantly shortened in the RH for both 2 and 10 semitones (significant main effect Hemisphere for N1 latency, $[\mathrm{F}(1,13)=6.093, \mathrm{p}<.05)])$.

The responses of $\mathrm{P} 1$ and $\mathrm{N} 1$ for 4 semitones and 10 semitones conditions from the first and second sessions of Experiment 1 (in adaptation and test position) were entered in another separate $2 \times 2 \times 2$ model ANOVA, in order to compare the effects induced by the preceding small $\Delta \mathrm{f}$ sequence (2 semitones). The statistical analysis showed that P1 was significantly increased in the LH for both $\Delta \mathrm{f}=4$ and $\Delta \mathrm{f}=10$ semitones conditions presented as Test sequence, compare to adaptation position (significant interaction for Hemisphere $\mathrm{x}$ Part $[F$ $(1,13)=11.747, p<.05])$. In contrast, the N1 amplitude was increased in the $\mathrm{RH}$ for these same conditions (significant main effect Hemisphere $[F(1,13)=6.759, p<.05]$ ). This lateralization effect was more prominent for $\Delta \mathrm{f}=10$ semitones during the Test sequence (significant interaction Hemisphere $\mathrm{x} \Delta \mathrm{f}[F(1,13)=8.851, p<.05]$, and $\Delta \mathrm{f} \mathrm{x}$ Part $[F(1,13)=8.044, p<.05])$. For the 4 and 10 semitones $\Delta \mathrm{f}$ stimuli presented only on adaptation position (no initial stimulation), significant effects were obtained regarding the lateralization of the amplitude of $\mathrm{N} 1$ (main effect Hemisphere $[F(1,14)=7.318, p<.05]$ and interaction Hemisphere $x \Delta f[F(1,14)=6.676, p<.05])$, the $\mathrm{N} 1$ was significantly larger in the $\mathrm{LH}$ for $\Delta \mathrm{f}=4$ semitones, whereas for $\Delta \mathrm{f}=10$ semitones the amplitude was stronger in the $\mathrm{RH}$.

A further separate ANOVA was applied to data from the first and second sessions in order to test the effects of an initial intermediate $(\Delta \mathrm{f}=4)$ and large $(\Delta \mathrm{f}=10)$ frequency separation on the small $\Delta \mathrm{f}$ Test condition. Only the amplitude of P1 was significantly enhanced when the 2 semitone stimulus was presented in the Test position condition compared to Adaptation position (significant main effect Part $[F(1,13)=6,331, \mathrm{p}<.05]$ ).

Figure 3 summarizes the fluctuations in neuronal activity and the lateralization effects for all $\Delta \mathrm{f}$ values $(2,4$ and 10 semitones), presented as Test or Adaptation, separately for the P1 and N1 components. Error bars indicate the 95\% confidence intervals for the within-subject effect (Part x Semitones), by Loftus \& Masson [46].

\section{Experiment 2. Influence of the alternating ISI}

The short ISI condition was analyzed in the timeinterval of $-50 \mathrm{~ms}$ to $400 \mathrm{~ms}$ and the long ISI condition was analyzed in the time-interval of $-50 \mathrm{~ms}$ to $800 \mathrm{~ms}$. The results of these analyses are displayed in Figure 4A with Adaptation and Test conditions denoted by black and grey lines, respectively. Concerning the B-tone, significant main effects and interaction regarding the position in the trial and the lateralization were neither obtained for P1 amplitude (main effect Hemisphere $[F(1,13)=1.397, p=0.258]$, ISI $[F(1,13)=2.036, p=0$ $.177]$, Part $[F(1,13)=3.201, p=0.097]$ and Hemisphere $\mathrm{x}$ Part $[F(1,13)=2.431, p=0.143])$ nor for N1 amplitude (main effect Hemisphere $[F(1,13)=2.024, p=0.178]$, ISI $[F(1,13)=0.604, p=0.451]$, Part $[F(1,13)=0.640, p=0$ $.438]$ and Hemisphere $x$ Part" $[F(1,13)=3.755, p=0$ $.075])$

Figure $4 \mathrm{~B}$ shows the neuronal activity and the lateralization effects for short ISI and long ISI, separately for P1 and N1 components, presented as Test or as Adaptation. Error bars represent 95\% confidence intervals for the within subject effect Part x Hemisphere [46].

\section{Behavioral data}

The results of the behavioral experiments are shown in Figure 5. The data obtained in response to small $\Delta \mathrm{f}$ (2 semitones) from the first two sessions were analyzed separately from the intermediate and large $\Delta \mathrm{f}$ conditions (4 and 10 semitones). The statistical analysis revealed significantly better performance of streaming when $\Delta \mathrm{f}$ of 4 and 10 semitones stimuli were presented in the Test position compared to the same stimuli presented in the Adaptation position (significant main effect Part [F $(1,11)=14.286, p<.05])$. In the third session no participants reported the perception of two separate streams in the long ISI condition, regardless of its presentation as Test or Adaptation sequence. In contrast, all participants perceived two streams in the short ISI condition but there was no significant difference related to the position as Test or Adaptation sequence $[t(11)=-1,149, p=0$. 275; independent sample $t$ test]. 


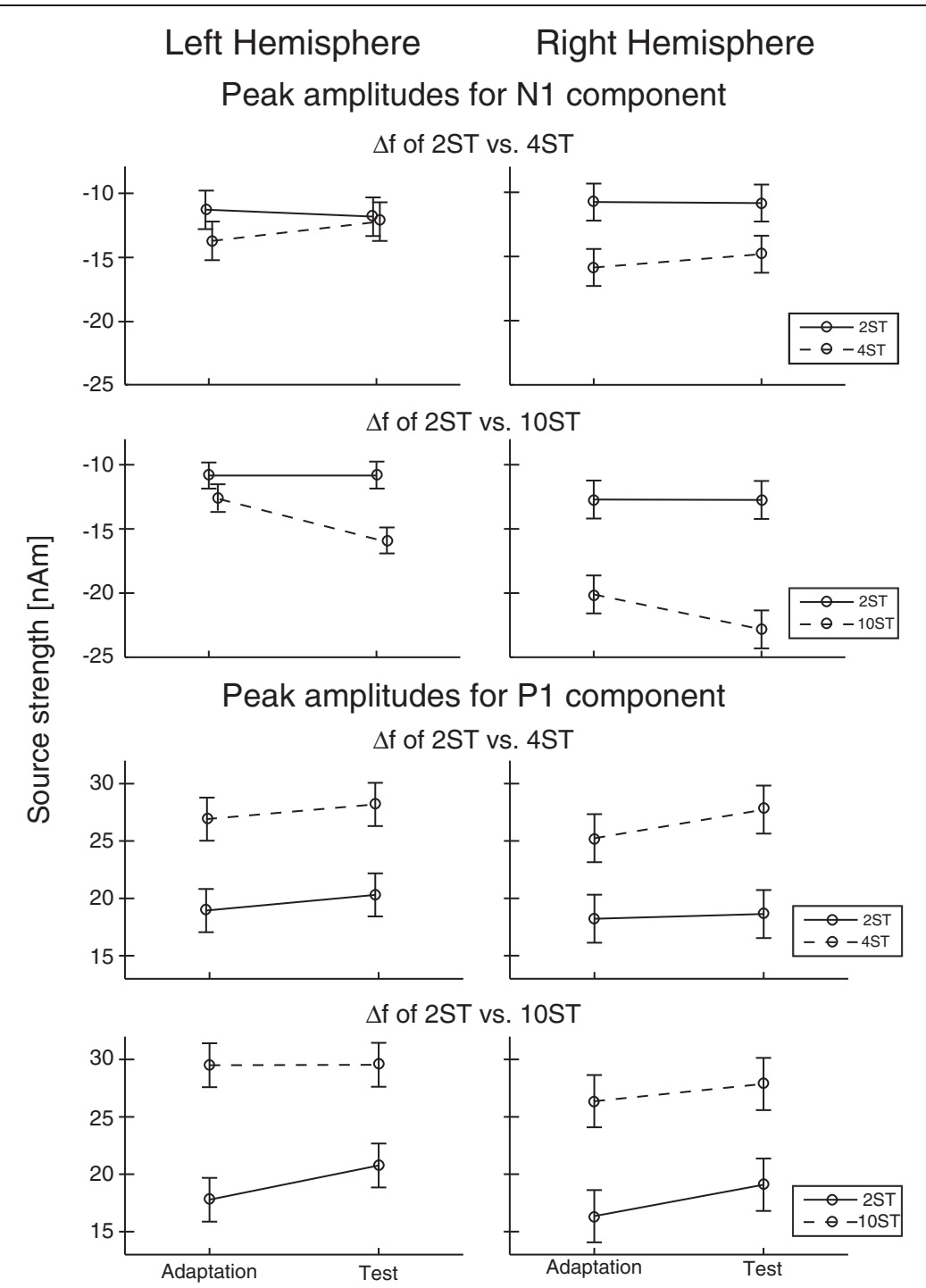

Figure 3 Peak amplitudes of $\mathbf{N} 1$ and P1 from each hemisphere to all three degrees of frequency separation. This figure shows responses tested across the two sessions which constituted Experiment 1 ( $\Delta f=2$ vs. 4 semtones; $\Delta f=2$ vs. 10 semitones) presented as Adaptation and Test sequence. Error bars represent 95\% confidence intervals. The upper plots show N1 responses and the lower plots show P1 responses.

\section{Discussion}

\section{Neurophysiologic correlates of stream segregation} Effect of alternating the frequency separation (Experiment 1)

To our knowledge, this study reports for the first time the differing behavior of $\mathrm{P} 1$ and $\mathrm{N} 1$ components of human AEFs when the frequency separation between A and $\mathrm{B}$ tones during initial Adaptation and subsequent Test sequences of a streaming task have alternated. Neural activity generally increased as the frequency separation between $\mathrm{A}$ and $\mathrm{B}$ tones increased, a finding which is in line with previous studies $[16,21,22,35]$. We found that in all conditions ( $\Delta \mathrm{f}$ of 2,4 and 10 semitones) the P1 enhancement was more prominent during the Test sequence but that the N1 amplitude was significantly increased only in the 10 semitone Test-condition.
Neuronal activity underlying streaming is known to be modified by prior adaptation [28,35], but the reported modulations of P1 and N1 components are not as specific, as seen here. Snyder and colleagues, for instance [35] performed a similar EEG study with a twoconsecutive-conditions design but with $1.44 \mathrm{~s}$ of silent interval between conditions. The $\Delta \mathrm{f}$ of the Adaptation sequence in their study was 3,6 , or 12 semitones, followed by a Test sequence with a $\Delta \mathrm{f}$ of 6 semitones. Unlike our design, the participants were required to pay attention to the stimuli. They reported that a small $\Delta \mathrm{f}$ in the Adaptation sequence induces P1 enhancement in response to the first A tone of the ABA triplet in the Test sequence. In the same manner, in our study the P1 component was enhanced in all conditions on a Test 


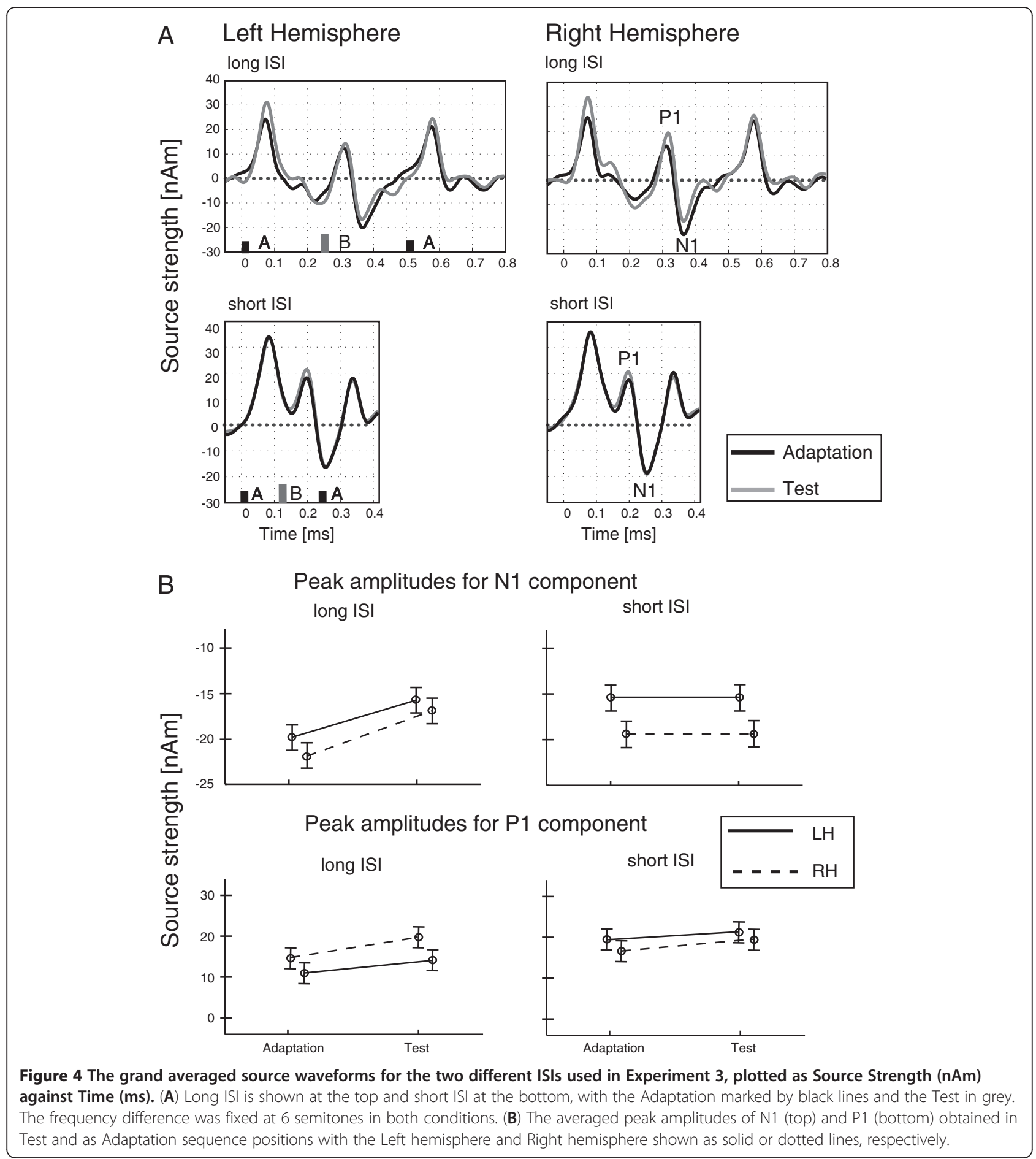

position, therefore the P1 clearly shows the context effect. We also found a significant enhancement of the N1 component in the case of the 10 semitone Test condition following the initial 2 semitones Adaptation sequence. This further modulation of the N1 could have been induced by the specific paradigm we used (two Adaptation and Test-sequences with no gap in between), because the contextual effect between consecutive tone patterns has been shown to depend upon the period of silence between them $[2,31,40,41]$ and to decrease as a function of time [35]. Furthermore, the enhancement of neural activity which we observed during the intermediate and large $\Delta \mathrm{f}$ Test sequences following adaptation to a small $\Delta f$ reliably concurred with our predictions the 


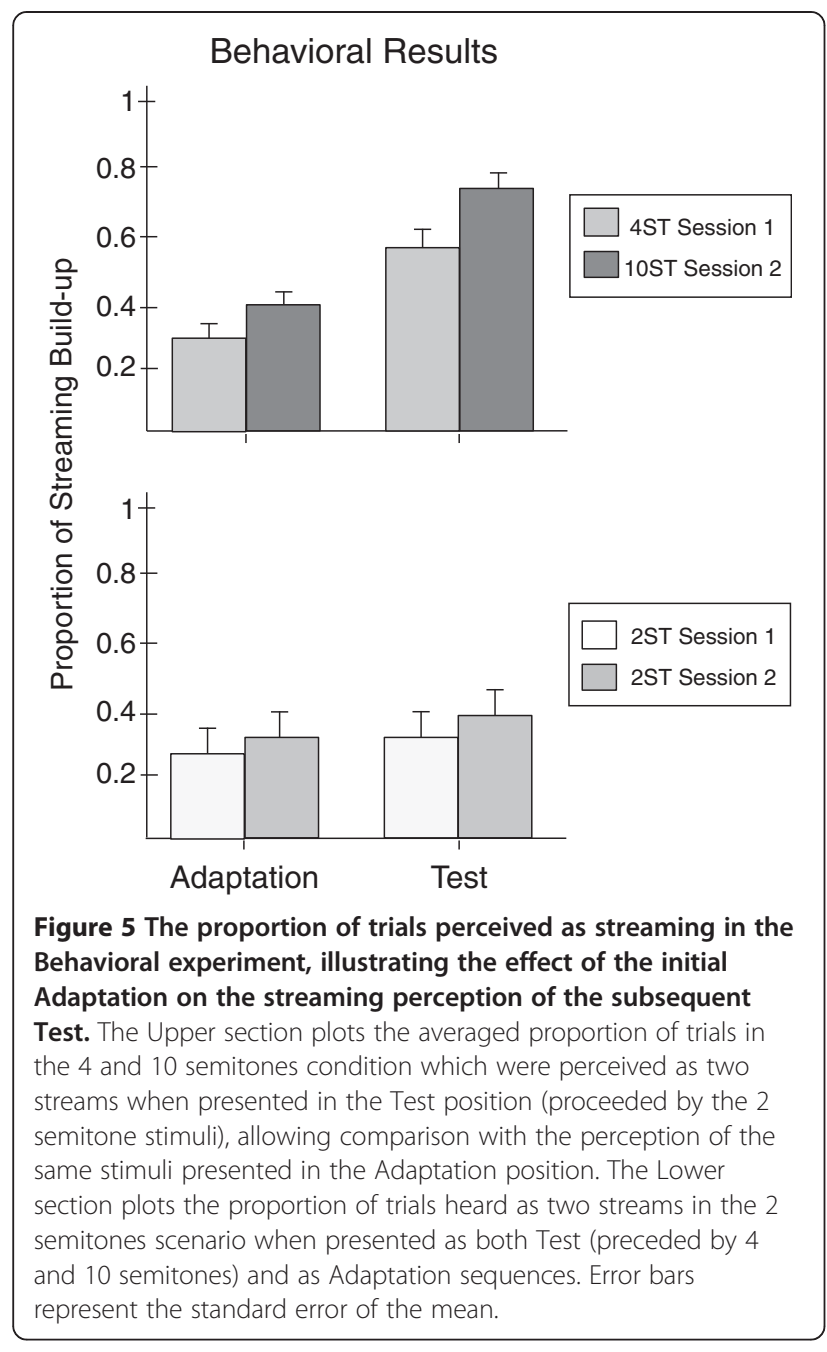

implications of previous research [35]. However, the amplitude of the P1 component was also found to increase in the small $\Delta \mathrm{f}$-Test sequence following initial larger $\Delta \mathrm{f}$ stimulation, despite our expectation that neuronal activity would reduce. The P1 component therefore appeared to be enhanced in all Test conditions, regardless of the size of the initial $\Delta \mathrm{f}$ Adaptation. This finding is contrary to previously reported psychophysical results [23] that demonstrated a reduced probability of two streams being perceived after adaptation to a larger $\Delta \mathrm{f}$ pattern. On the other hand, Snyder and colleagues $[23,34]$ proposed that multiple levels of representation could underlie the perceptual organization of sequential auditory patterns, and the facilitative perceptual effect and the stimulus driven-effect are proposed to be at least partially independent [34]. It has also been suggested that distinct cortical areas may be active in the encoding of the adaptation and test streaming patterns [35]. Given this suggestion, it is likely that the P1 and N1 response components represent different modulation effects in the encoding of subsequent streaming patterns: P1 demonstrates the adaptation effect (context) and N1 demonstrates the streaming effect. Further support for this assumption is in the dissimilar task sensitivity of both components [47], and this is in turn supported by evidence that P1 and N1 are generated by different auditory cortical regions [48-51]. Moreover, distinct neuronal mechanisms are responsible for automatic auditory stream segregation and attention-dependent buildup processes $[8,22,28,38]$, and attention operates in a more pronounced manner on $\mathrm{N} 1$ and later components (P2 and N2) [36,51-53]. For this reason, additional modulation in the auditory cortical network could be expected in an active listening task.

\section{Lateralization effects, based on frequency alternations}

Our results from the first experiment showed lateralizationdifferences of the B tone-related P1 and N1 components between different frequency separation conditions (2, 4 and 10 semitones). The amplitude of the P1 component was significantly increased for all conditions ( $\Delta \mathrm{f}$ of 2,4 and 10 semitones) in the LH during the Test sequence. In contrast, the $\mathrm{N} 1$ amplitude was significantly enhanced in the RH for higher frequency separations only ( $\Delta \mathrm{f}$ of 4 and 10 semitones). The N1 latency was also shortened in the case of the 10 semitones stimulus in the Test, which is in line with the findings of Roberts \& Poeppel who demonstrated that the N1 peak latency varies as a function of the stimulus frequency [54]. It has been also proposed that amplitude and latency lateralization of the N1 component has a common origin [55]; a proposal which can also be observed in our data ( $\mathrm{RH}$ amplitude and latency lateralization of N1). Regarding streaming, Snyder and colleagues demonstrated that the modulation of N1 occurred at the right but not at the left temporal electrode, suggesting an $\mathrm{RH}$ dominance for frequency separation-based segregation [22], in line with our findings.

In summary, the differences in the modulation of the AEFs that were demonstrated in the first experiment suggest that P1 and N1 represent at least partiallydifferent mechanisms both of which are active during the automatic segregation of subsequent streaming patterns. The two components also showed different lateralization behavior: P1 was commonly enhanced in the LH in all conditions, whereas N1 was increased significantly only in the RH during the 10 semitone Test sequence.

\section{Effect of alternating the ISI (Experiment 2)}

The goal of the second experiment was to test the effect of alternating the ISI between A and B tones in consecutive Adaptation and Test conditions in an uninterrupted trial. The frequencies of $\mathrm{A}$ and $\mathrm{B}$ tones were kept constant during the whole trial; therefore the tonotopic 
representation was identical. The morphology of the AEF responses to the short ISI condition in both the Test and Adaptation positions was almost identical and not significantly different in comparison to long ISI responses. Thus, the presentation of an initial long ISI condition did not influence the segregation process during the following short ISI sequence and vice versa. Since adaptation in the auditory cortex is dependent not only on the specific frequency but also on the concrete repetition rate of the stimuli $[13,56,57]$, it could be suggested that the different ISIs which were used in the consecutive Adaptation and Test sequences caused disruption of the frequency-specific adaptation to $\mathrm{A}$ and $\mathrm{B}$ tones. Both sequences were therefore processed as distinct temporal events, without the contextual influence of the neural activity of one upon the other, regardless of having the same tonotopic representation. Based on that finding, it could be suggested that the modulation effects reported in the first experiment reflect the repeated presentation of specific tone frequencies but not of neurons tuned to a certain $\Delta \mathrm{f}$-shift [3]. This idea has also been tested in a recent psychophysical study by using Adaptation sequence A tones of greatly different frequency to those of the Test sequence, and B tones of either 3 or 12 semitones above the A tone frequency [23]. The presentation of a small or large $\Delta f$ initial sequence did not affect the perception of streaming during the test, supporting the idea that the context effect reflects adaptation to the repeated presentation of specific tone frequencies [23]. It should be mentioned, however, that the ISI values used in the present experiment were extreme (one very long ISI and one half the length) and that these conclusions regarding the concrete ISIs and modulation of the neural activity are therefore not necessarily applicable to other ISI conditions.

\section{Behavioral study}

The behavioral study was conducted in order to evaluate the participants' perception using the same paradigm as in the MEG measurements. All aspects of the previous MEG experiments were replicated as well as the behavioral measurements. The results revealed that the likelihood of reporting two streams increased as $\Delta \mathrm{f}$ increased, a finding which is in line with previous studies $[16,21,22,35,39]$. The main purpose was to evaluate the context effects of initial adaptation on the streaming perception during the Test sequence, in order to compare them with the neurophysiological findings. The results demonstrated a greater probability perceptual streaming in response to large and intermediate $\Delta \mathrm{f}$ in the Test sequence position following a small $\Delta \mathrm{f}$ Adaptation sequence compared with a large or intermediate $\Delta f$ on Adaptation position. No significant effects or interactions were found regarding the perception of small $\Delta f$
Test sequence when preceded by large (10 semitones) and intermediate ( 4 semitones) $\Delta \mathrm{f}$ Adaptation presentations despite the fact that the P1 component appeared to be enhanced in the MEG outcome of our study. The lack of perceptual modulation during the 2 semitone Test sequence could have been caused by using a small $\Delta \mathrm{f}$ because the perceptual context effect was reported to be strongest at intermediate $\Delta f$ values [23,34,35]. Furthermore, it has been proposed that streaming build-up occurs at levels of neuronal representation with sharp frequency tuning, unlike the effect of prior context which is caused by the adaptation of neurons with wide frequency tuning [23]. Thus, modulations in neural activity were reported in our neurophysiological experiments in all Test conditions (enhanced P1 component), unlike the perceptual effect which was less responsive to the initial large $\Delta \mathrm{f}$ Adaptation conditions.

The behavioral data obtained when altering the ISI, showed no significant differences in the short ISI Test condition when preceded by the long ISI Adaptation sequence and vice-versa. We therefore conclude that adaptation sequences of different ISIs do not affect the subsequent perception of streaming. This psychophysical finding compliments the results of the corresponding MEG experiment which showed no AEF modulation following variation of ISI in the Adaptation sequence. As proposed above, the neurons undergoing the adaptation effect are sensitive not only to a specific frequency range, but also to the concrete repetition rate of the presenting stimuli $[13,56,57]$. Our findings lend further support to the idea that the initial adaptation that facilitates the streaming process is rather a peripheral mechanism that depends on simple tone frequencies but not higher order auditory features [23].

In addition, it should be mentioned that the perceptual state cannot be directly ascribed to the electrophysiological data seen in prior pre-attentive MEG experiments, since the buildup of streaming requires several seconds [2,31]. Moreover, different levels of representation underlying the contextual influence in auditory stream segregation have been suggested by Snyder and colleagues [23] as pointed out above, and the effect of the initial context that facilitates the streaming perception appeared to be different from the neural effect caused by the prior small or large $\Delta \mathrm{f}$-based adaptation $[28,35]$.

\section{Conclusions}

Our MEG experiment demonstrates dissimilar behavior in P1 and N1 response components during the automatic segregation of sound, induced by an initial Adaptation sequence. The P1 component appeared to be enhanced in all Test conditions and thus demonstrates the preceding context effect, whereas N1 was specifically 
modulated only in the large frequency separation Test condition induced by a preceding small $\Delta \mathrm{f}$ Adaptation sequence. This finding combined with the difference we observed in the lateralization of P1 and N1 could suggest that both components represent at least partiallydifferent systems underlying the perceptual organization of streaming patterns. The psychophysical results of the parallel behavioral study show that prior adaptation to a smaller degree of frequency separation facilitates the streaming perception. However, neither the neural activity nor the perception of successive streaming sequences were modulated when the inter stimulus intervals were varied, thus lending support to the idea that the initial modulation effect reflects adaptation to the repeated presentation of specific tone-frequencies.

\section{Competing interests}

The authors declare that they have no financial or any other competing interests.

\section{Authors' contributions}

IC, RD and CP conceived of the study designed the experimental setup and the auditory stimuli. IC and RD acquired the data. IC performed the data \& statistical analyses. All authors participated the data evaluation and interpretation and in writing the manuscript, and have approved the final version of the manuscript.

\section{Acknowledgements}

We are grateful to Maximilian Bruchmann for discussions on the statistical analysis, to Ross Parfitt for the helpful comments and Karin Berning for technical assistance. This work has been supported by "Deutsche Forschungsgemeinschaft", (DR 807/1-1).

\section{Author details}

${ }^{1}$ Institute for Biomagnetism and Biosignalanalysis, University of Münster, Malmedyweg 15, 48149, Münster, Germany. ${ }^{2}$ MEG-Center, Eberhard-KarlsUniversity Tübingen, Otfried-Müller-Straße 47, 72076, Tübingen, Germany.

Received: 11 February 2012 Accepted: 21 May 2012

Published: 20 June 2012

\section{References}

1. Bregman AS, Campbell J: Primary auditory stream segregation and perception of order in rapid sequences of tones. J Exp Psychol 1971, 89(2):244-249.

2. Bregman AS: Auditory streaming is cumulative. J Exp Psychol Hum Percept Perform 1978, 4(3):380-387.

3. Van Noorden L: Temporal cocherence in the perception of tone sequences. In Eindhoven. the Niederlands: Eindhoven University of Technology; 1975.

4. Zwislocki J: Theory of temporal auditory summation. J Acoust Soc Am 1960, 32:1046-1059.

5. Pressnitzer D, Hupe JM: Temporal dynamics of auditory and visual bistability reveal common principles of perceptual organization. Curr Biol 2006, 16(13):1351-1357.

6. Carlyon RP, Cusack R, Foxton JM, Robertson $\mathrm{H}$ : Effects of attention and unilateral neglect on auditory stream segregation. J Exp Psychol Hum Percept Perform 2001, 27(1):115-127.

7. Hartmann WM JD: Sream segregation and peripheral channeling. Music Perception 1991, 9:155-184.

8. Carlyon RP: How the brain separates sounds. Trends Cogn Sci 2004 8(10):465-471.

9. Carlyon RP, Micheyl C, Deeks JM, Moore BC: Auditory processing of real and illusory changes in frequency modulation (FM) phase. J Acoust SOC Am 2004, 116(6):3629-3639.

10. Carlyon RP, Plack CJ, Fantini DA, Cusack R: Cross-modal and non-sensory influences on auditory streaming. Perception 2003, 32(11):1393-1402.
11. Gockel H, Moore BC, Patterson RD: Asymmetry of masking between complex tones and noise: the role of temporal structure and peripheral compression. J Acoust Soc Am 2002, 111(6):2759-2770.

12. Bee MA, Klump GM: Auditory stream segregation in the songbird forebrain: effects of time intervals on responses to interleaved tone sequences. Brain Behav Evol 2005, 66(3):197-214.

13. Brosch $M$, Schreiner CE: Time course of forward masking tuning curves in cat primary auditory cortex. J Neurophysio/ 1997, 77(2):923-943.

14. Fishman Yl, Arezzo JC, Steinschneider M: Auditory stream segregation in monkey auditory cortex: effects of frequency separation, presentation rate, and tone duration. J Acoust Soc Am 2004, 116(3):1656-1670.

15. Fishman Yl, Reser DH, Arezzo JC, Steinschneider M: Neural correlates of auditory stream segregation in primary auditory cortex of the awake monkey. Hear Res 2001, 151(1-2):167-187.

16. Gutschalk A, Micheyl C, Melcher JR, Rupp A, Scherg M, Oxenham AJ: Neuromagnetic correlates of streaming in human auditory cortex. J Neurosci 2005, 25(22):5382-5388.

17. Kanwal JS, Medvedev AV, Micheyl C: Neurodynamics for auditory stream segregation: tracking sounds in the mustached bat's natural environment. Network 2003, 14(3):413-435.

18. Micheyl C, Tian B, Carlyon RP, Rauschecker JP: Perceptual organization of tone sequences in the auditory cortex of awake macaques. Neuron 2005, 48(1):139-148

19. Micheyl C, Carlyon RP, Gutschalk A, Melcher JR, Oxenham AJ, Rauschecker JP, Tian B, Courtenay Wilson E: The role of auditory cortex in the formation of auditory streams. Hear Res 2007, 229(1-2):116-131.

20. Snyder JS, Alain C: Sequential auditory scene analysis is preserved in normal aging adults. Cereb Cortex 2007, 17(3):501-512.

21. Snyder JS, Alain C: Age-related changes in neural activity associated with concurrent vowel segregation. Brain Res Cogn Brain Res 2005, 24(3):492-499.

22. Snyder JS, Alain C, Picton TW: Effects of attention on neuroelectric correlates of auditory stream segregation. J Cogn Neurosci 2006, 18(1):1-13.

23. Snyder JS, Carter OL, Hannon EE, Alain C: Adaptation reveals multiple levels of representation in auditory stream segregation. J Exp Psychol Hum Percept Perform 2009, 35(4):1232-1244.

24. Wehr M, Zador AM: Synaptic mechanisms of forward suppression in rat auditory cortex. Neuron 2005, 47(3):437-445.

25. Ulanovsky N, Las L, Nelken I: Processing of low-probability sounds by cortical neurons. Nat Neurosci 2003, 6(4):391-398.

26. Schadwinkel S, Gutschalk A: Activity associated with stream segregation in human auditory cortex is similar for spatial and pitch cues. Cereb Cortex 2010, 20(12):2863-2873.

27. Schadwinkel S, Gutschalk A: Transient bold activity locked to perceptual reversals of auditory streaming in human auditory cortex and inferior colliculus. J Neurophysio/ 2011, 105(5):1977-1983.

28. Sussman E, Steinschneider M: Neurophysiological evidence for contextdependent encoding of sensory input in human auditory cortex. Brain Res 2006, 1075(1):165-174.

29. Kondo HM, Kashino M: Involvement of the thalamocortical loop in the spontaneous switching of percepts in auditory streaming. J Neurosci 2009, 29(40):12695-12701.

30. Beauvois MW, Meddis R: Computer simulation of auditory stream segregation in alternating-tone sequences. J Acoust Soc Am 1996, 99(4 Pt 1):2270-2280.

31. Beauvois MW, Meddis R: Time decay of auditory stream biasing. Percept Psychophys 1997, 59(1):81-86.

32. Pressnitzer D, Sayles M, Micheyl C, Winter IM: Perceptual organization of sound begins in the auditory periphery. Curr Biol 2008, 18(15):1124-1128.

33. Elhilali M, Ma L, Micheyl C, Oxenham AJ, Shamma SA: Temporal coherence in the perceptual organization and cortical representation of auditory scenes. Neuron 2009, 61(2):317-329.

34. Snyder JS, Carter OL, Lee SK, Hannon EE, Alain C: Effects of context on auditory stream segregation. J Exp Psychol Hum Percept Perform 2008, 34(4):1007-1016.

35. Snyder JS, Holder WT, Weintraub DM, Carter OL, Alain C: Effects of prior stimulus and prior perception on neural correlates of auditory stream segregation. Psychophysiology 2009, 46(6):1208-1215.

36. Hillyard SA, Hink RF, Schwent VL, Picton TW: Electrical signs of selective attention in the human brain. Science 1973, 182(108):177-180. 
37. Hillyard SA: Electrical and magnetic brain recordings: contributions to cognitive neuroscience. Curr Opin Neurobiol 1993, 3(2):217-224.

38. Cusack R, Deeks J, Aikman G, Carlyon RP: Effects of location, frequency region, and time course of selective attention on auditory scene analysis. J Exp Psychol Hum Percept Perform 2004, 30(4):643-656.

39. Snyder JS, Alain C: Toward a neurophysiological theory of auditory stream segregation. Psychol Bull 2007, 133(5):780-799.

40. Bregman AS, Ahad PA, Crum PA, O'Reilly J: Effects of time intervals and tone durations on auditory stream segregation. Percept Psychophys 2000, 62(3):626-636.

41. Rogers WL, Bregman AS: An experimental evaluation of three theories of auditory stream segregation. Percept Psychophys 1993, 53(2):179-189.

42. Cowan N: On short and long auditory stores. Psychol Bull 1984, 96:341-370.

43. Grossheinrich N, Kademann S, Bruder J, Bartling J, Von Suchodoletz W: Auditory sensory memory and language abilities in former late talkers: a mismatch negativity study. Psychophysiology 2010, 47(5):822-830.

44. Copeland D, Radvansky G: Working memory and syllogistic reasoning. Q J Exp Psychol A 2004, 57(8):1437-1457.

45. Tesche CD, Uusitalo MA, IImoniemi RJ, Huotilainen M, Kajola M, Salonen O: Signal-space projections of MEG data characterize both distributed and well-localized neuronal sources. Electroencephalogr Clin Neurophysiol 1995, 95(3):189-200.

46. Loftus GR, Masson MEJ: Using confidence intervals in within-subject designs. Psychon Bull Rev 1994, 47(1):476-490.

47. Chait M, Simon JZ, Poeppel D: Auditory M50 and M100 responses to broadband noise: functional implications. Neuroreport 2004, 15(16):2455-2458.

48. McEvoy L, Makela JP, Hamalainen M, Hari R: Effect of interaural time differences on middle-latency and late auditory evoked magnetic fields. Hear Res 1994, 78(2):249-257.

49. Makela JP, Hamalainen M, Hari R, McEvoy L: Whole-head mapping of middle-latency auditory evoked magnetic fields. Electroencephalogr Clin Neurophysiol 1994, 92(5):414-421.

50. Levanen S, Sams M: Disrupting human auditory change detection: Chopin is superior to white noise. Psychophysiology 1997, 34(3):258-265.

51. Pantev C, Okamoto H, Ross B, Stoll W, Ciurlia-Guy E, Kakigi R, Kubo T: Lateral inhibition and habituation of the human auditory cortex. Eur J Neurosci 2004, 19(8):2337-2344

52. Coenen A: Modelling of auditory evoked potentials of human sleep-wake states. Int J Psychophysiol 2011, :-. In press.

53. Tian S, Qi H, Wang J, Cai J, Ma Y: Differential amplitude modulation of auditory evoked cortical potentials associated with brain state in the freely moving rhesus monkey. Neurosci Lett 2002, 331(3):159-162.

54. Roberts TP, Poeppel D: Latency of auditory evoked M100 as a function of tone frequency. Neuroreport 1996, 7(6):1138-1140.

55. Howard MF, Poeppel D: Hemispheric asymmetry in mid and long latency neuromagnetic responses to single clicks. Hear Res 2009, 257(1-2):41-52.

56. Calford MB, Semple MN: Monaural inhibition in cat auditory cortex. J Neurophysiol 1995, 73(5):1876-1891.

57. Draganova R, Ross B, Borgmann C, Pantev C: Auditory cortical response patterns to multiple rhythms of AM sound. Ear Hear 2002, 23(3):254-265.

doi:10.1186/1471-2202-13-72

Cite this article as: Chakalov et al:: Modulations of neural activity in auditory streaming caused by spectral and temporal alternation in subsequent stimuli: a magnetoencephalographic study. BMC Neuroscience 2012 13:72.

\section{Submit your next manuscript to BioMed Central and take full advantage of:}

- Convenient online submission

- Thorough peer review

- No space constraints or color figure charges

- Immediate publication on acceptance

- Inclusion in PubMed, CAS, Scopus and Google Scholar

- Research which is freely available for redistribution 